\title{
The Amino Acid Utilization by Phase I Bordetella pertussis in a Chemically Defined Medium
}

\author{
BY M. GOLDNER, CVETKA M. JAKUS, HARRIETT K. RHODES \\ AND R. J. WILSON \\ Connaught Medical Research Laboratories, University of Toronto, Toronto, Canada
}

(Received 28 February 1966)

\begin{abstract}
SUMMARY
Phase I Bordetella pertussis was grown in a chemically defined medium containing 20 amino acids (including glutamine) plus glutathione. Growth was limited by depletion of three of the components, L-glutamic acid, L-proline and L-glutamine. Increasing the concentrations of these three components more than doubled the yield of organism. The following components were utilized, but did not limit growth: alanine, glycine, histidine, serine; cystine and glutathione were also substantially used. Ten amino acids which were not utilized to any significant extent could be omitted without diminishing growth. A preferential order of amino acid utilization is suggested. Glutamic acid appears to be important for antigenicity.
\end{abstract}

\section{INTRODUCTION}

There has been some interest in propagating phase I Bordetella pertussis in defined media. Rowatt $(1957$ ) reviewed the growth requirements of this micro-organism and Wilson (1963) cultivated it in a chemically defined medium. The present investigation concerns the various amino acids used in the growth of $B$. pertussis phase I in Wilson's (1963) medium. Based on these utilization results, the amino acid composition of the medium was modified; the resulting changes in yield of organisms, utilization of amino acids and antigenicity are reported.

\section{METHODS}

Medium, inoculum and growth conditions. Table 1 lists the amounts of amino acids (and glutamine) and glutathione present in the medium (Wilson, 1963). In addition, this medium contains inorganic salts, nucleic acid derivatives, vitamins, adenosine triphosphate and coenzymes. The medium is defined except for the presence of a liver coenzyme concentrate which contains coenzyme A, NADP and NAD. This liver coenzyme concentrate can be replaced with equivalent amounts of these coenzymes without loss of growth of Bordetella pertussis. This chemically defined medium is designated medium $\mathbf{A}$.

Modifications of medium $A$ with respect to the presence and concentration of amino acids.

Medium B contained a fourfold increase in L-glutamic acid, L-glutamine and L-proline.

Medium C contained a fourfold increase in L-glutamic acid only. 
Medium D. Hydroxyproline, isoleucine, leucine, lysine, methionine, phenylalanine, threonine, tryptophan, tyrosine and valine were omitted from medium $A$.

Medium $\mathrm{E}$. The same amino acids were omitted as in medium $\mathrm{D}$, and the concentration of L-glutamic acid, L-glutamine and L-proline quadrupled.

Medium F. The same amino acids were omitted as in medium $\mathbf{D}$, and the concentration of L-glutamic acid only quadrupled.

The inoculum (Siebenmann strain, Connaught Medical Research Laboratories) consisted of $40 \times 10^{6}$ organisms $/ \mathrm{ml}$. medium (Wilson, 1963). The cultures were grown in $50 \mathrm{ml}$. volumes in L-shaped tubes, which were rocked gently at $35-36^{\circ}$ for $48 \mathrm{hr}$.

\section{Table 1. Amino acids present in chemically defined medium for growth of phase I Bordetella pertussis (Wilson, 1963)}

$\begin{array}{lrlr} & \text { (mg./1.) } & & \text { (mg./l.) } \\ \text { DL-alanine } & 100 & \text { DL-methionine } & 60 \\ \text { L-arginine } & 40 & \text { DL-phenylalanine } & 100 \\ \text { DL-aspartic acid } & 130 & \text { L-proline } & 60 \\ \text { L-cystine } & 10 & \text { DL-serine } & 130 \\ \text { L-glutamic acid } & 155 & \text { DL-threonine } & 80 \\ \text { Glycine } & 50 & \text { DL-tryptophan } & 30 \\ \text { DL-histidine } & 50 & \text { L-tyrosine } & 40 \\ \text { L-hydroxyproline } & 20 & \text { DL-valine } & 120 \\ \text { DL-isoleucine } & 120 & \text { L-glutamine } & 100 \\ \text { DL-leucine } & 240 & \text { Glutathione (red.) } & 25 \\ \text { L-lysine } & 70 & & \end{array}$

Bacterial concentrations were measured at $530 \mathrm{~m} \mu$ (Coleman Universal Spectrophotometer, model 14) and the growth determined by relating the turbidity reading to the number of organisms $/ \mathrm{ml}$. based on the National Institutes of Health U.S. Standard (10 opacity units).

Amino acid analysis of culture media by paper chromatography. Fresh media and culture filtrates were concentrated in vacuum sixfold and the amino acids separated by one-dimensional paper chromatography on Whatman no. 3 filter paper $(88 \times 5 \mathrm{~cm}$.) for $46 \mathrm{hr}$ at $24^{\circ} \pm 1$. The solvent system was $n$-propanol + ethanol $+0.05 \mathrm{M}$-pyrophosphate buffer ( $\mathrm{pH} 8 \cdot 9)$ + water $(55+22 \cdot 5+10+12 \cdot 5$, by vol.) containing $0 \cdot 22 \%$ (w/v) NaCl (Wade, Matheson \& Hanes, 1961; Hanes, Harris, Moscarello \& Tigane, 1961).

One-dimensional paper chromatography was also done with solvent $n$-butanol + acetic acid + water $(4+1+5$, by vol.; Slotta $\&$ Primosigh, 1951) on Whatman no. 3 paper $\left(57 \times 46 \mathrm{~cm}\right.$.) for $66 \mathrm{hr}$ at $24^{\circ} \pm 1$.

The chromatograms were sprayed with ninhydrin (Block, 1950) and kept in the dark for $24 \mathrm{hr}$. Those developed in the $n$-propanol system were assayed in a Photovolt automatic densitometer. Preliminary determinations with this instrument established the relation, ' $\mathrm{area}^{2}$ varies as concentration of amino acid' on the basis of a statistical analysis (Block, Durrum \& Zweig, 1958; D. B. W. Reid \& G. Van Belle, personal communication). By this procedure, the error in the determination was of the order of $15 \%$.

Antigenic studies. For antigenic studies, vaccines were prepared from formalininactivated cultures after incubation for $48 \mathrm{hr}$ in the various media. The vaccines were assayed by the mouse protection test, and their potencies determined as 
immunogenic units against the U.S. Standard Pertussis Vaccine according to the Wilson-Worcester statistical method (Worcester \& Wilson, 1943).

\section{RESULTS}

In the results given below, the terms 'use' or 'utilization' relate to the disappearance or decrease in intensity of spots on the chromatograms.

Cultures of Bordetella pertussis in medium A reached a maximum population of $5 \times 10^{9}$ organisms $/ \mathrm{ml}$. by $48 \mathrm{hr}$. Where only L-isomers or the DL-mixture of the amino acids were present in the medium, the same maximum concentration of organisms was reached, but only when the concentration of the DL-mixture forms were doubled when replacing the $\mathrm{L}$-forms in the original medium A. Otherwise, the growth was less.

Many of the amino acids present in medium $\mathbf{A}$ could be separated chromatographically in the $n$-propanol system. However, certain spots consisting of two amino acids were separable in the $n$-butanol system. Thus cystine, when separated from aspartic acid, was seen to be substantially utilized. It was also possible to separate tyrosine from tryptophan, methionine from valine and hydroxyproline from threonine; serine and glycine, however, remained together. Utilization of tyrosine and tryptophan occurred, but no utilization of methionine. The decrease in intensity of the spots of these particular components was estimated visually except for cystine whose utilization was measured with a manual densitometer (EEL densitometer).

Determinations of amino acid utilization from medium $\mathbf{A}$ are shown in Table 2. The $\%$ utilization results were expressed as scores from 0 to 3 . (The score of 0 assigned to some DL-amino acids indicates that little or no utilization occurred even when the L-form of the amino acid was considered to be the only utilizable one.)

Since glutamic acid, proline and glutamine at the initial concentrations used were fully depleted during growth of Bordetella pertussis, the concentrations of these components were increased in subsequent experiments. Quadrupling the concentrations of L-glutamic acid, L-proline and L-glutamine (medium B) increased the yield of organisms in $48 \mathrm{hr}$ by $100-130 \%$. There was no indication that any significant change in the yield of organisms could be expected after further increases of these components to the medium. When L-glutamic acid alone was quadrupled (medium C), 70-90\% more growth was obtained than with medium A.

Densitometric determinations of the chromatograms for these media are given in Table 2. Glutamic acid, proline, glutamine, serine-glycine and alanine were considerably or completely utilized; glutathione was substantially used. The histidine + lysine mixture seemed in some instances to be used less than in the original medium; the cystine + aspartic acid mixture was always moderately used.

Ten amino acids (hydroxyproline, isoleucine, leucine, lysine, methionine, phenylalanine, threonine, tryptophan, tyrosine, valine) which showed little or no evidence of utilization following growth in medium $\mathbf{A}$ were omitted (medium D) without a decrease in growth. Quadrupling the concentrations of L-glutamic acid, L-proline and L-glutamine (medium E), or of L-glutamic acid alone (medium F), resulted in increased $48 \mathrm{hr}$ growths of 90-125\% and 60-105\%, respectively, as compared with that in medium A. Densitometric analysis of chromatograms of these media with 
the ten amino acids omitted showed the same complete or extensive utilization of glutamic acid, proline, glutamine, serine + glycine and alanine as occurred in media A, B and C (see Table 2). Glutathione was also used extensively, while glutamine appeared to be slightly less utilized than in medium A. Histidine was utilized to a

\section{Table 2. Utilization of amino acids in the growth of phase I Bordetella pertussis in chemically defined media}

Incubation $48 \mathrm{hr}$ at $37^{\circ}$. Amino acid content of concentrated coelum fluids estimated chromatographically (see text).

\begin{tabular}{|c|c|c|c|c|c|}
\hline \multicolumn{6}{|c|}{ Score* } \\
\hline \multicolumn{3}{|c|}{ Medium $\dagger$} & \multicolumn{3}{|c|}{ Medium $\dagger \ddagger$} \\
\hline A & B & C & D & $\mathbf{E}$ & $\mathbf{F}$ \\
\hline 3 & 3 & 3 & 3 & 3 & 3 \\
\hline $\mathbf{0}$ & 0 & 0 & 0 & 0 & 0 \\
\hline 1 & - & - & - & - & - \\
\hline 2 & - & - & - & - & - \\
\hline- & 1 & 1 & 1 & 1 & 1 \\
\hline $\mathbf{3} \|$ & 3 & 3 & 3 & 3 & 3 \\
\hline 3 & - & - & - & - & - \\
\hline- & 3 & 3 & 3 & 3 & $\mathbf{3}$ \\
\hline- & 3 & 3 & 3 & 3 & 3 \\
\hline 2 & 2 & 2 & 2 & 2 & 1 \\
\hline $\mathbf{3} \|$ & 3 & 3 & 3 & 3 & 3 \\
\hline $\mathbf{3} \|$ & $\mathbf{3}$ & 29 & 3 & 3 & 3 \\
\hline 3 & 39 & 2 & 3 & 3 & 3 \\
\hline 0 & 0 & 0 & & & \\
\hline 0 & - & () & & & \\
\hline 0 & - & 0 & & & \\
\hline- & 1 & - & & & \\
\hline 0 & 0 & 0 & & & \\
\hline 0 & - & - & & & \\
\hline 0 & - & - & & & \\
\hline- & 1 & 1 & & & \\
\hline $\mathbf{1}$ & 1 & 0 & & & \\
\hline 0 & $\mathbf{0}$ & 0 & & & \\
\hline 1 & 1 & $\mathbf{2}$ & & & \\
\hline 5 & $\left\{\begin{array}{l}10- \\
11 \cdot 5\end{array}\right.$ & $\left.\begin{array}{l}8 \cdot 5- \\
9 \cdot 5\end{array}\right\}$ & 5 & $\left\{\begin{array}{r}9.5 \\
11.25\end{array}\right.$ & $\begin{array}{c}8- \\
10 \cdot 25\end{array}$ \\
\hline
\end{tabular}

(organisms $\times 10^{-9} / \mathrm{ml}$.)

Alanine

Arginine

Aspartic acid

Cystine

Cystine§ + aspartic acid

Glutamic acid

Serine + glycine

Glycine

Serine

Histidine

Proline

Glutamine

Glutathione

Hydroxyproline

Isoleucine

Leucine

Isoleucine + leucine

Lysine

Methionine

Valine

Methionine + valine

Phenylalanine

Threonine

Tyrosine + tryptophan

Growth after $48 \mathrm{hr}$

Utilization after $48 \mathrm{hr}$

Between 0 and $15 \%$

Between 16 and $45 \%$

Between 46 and $75 \%$

Between 76 and $100 \%$

Components are listed as present in each medium (see text under 'Methods' for details).

$\ddagger$ Ten amino acids present in medium $A$ were omitted.

$\S$ Not assayed separately.

\| Most utilized components in medium A.

I Could score either 2 or 3 .

substantial extent, as in medium A. Aspartic acid was moderately used, even in respect to the L-form only.

Vaccines were prepared from cultures grown in all the media described. The results of mouse protection tests showed that media $\mathbf{B}$ and $\mathbf{C}$ produced more immunogenic vaccines than did medium A (Table 3). Antigenicity tests also indicated 
that the vaccines prepared from cultures grown in the other media $(D, E, F)$ were in some cases more antigenic than those prepared from cultures in medium $\mathbf{A}$.

Table 3. Vaccine potency of phase I Bordetella pertussis cultures from regular and modified chemically defined media

\begin{tabular}{|c|c|}
\hline $\begin{array}{l}\text { Vaccine prepared } \\
\text { in medium }\end{array}$ & $\begin{array}{r}\text { Potenc) } \\
\text { (immunogenic }\end{array}$ \\
\hline $\mathbf{A}$ & $\begin{array}{l}1 \cdot 5 \\
2 \cdot 0\end{array}$ \\
\hline $\mathbf{B}$ & $\begin{array}{l}4 \cdot 6 \\
4 \cdot 1 \\
8 \cdot 0\end{array}$ \\
\hline C & $\begin{array}{l}7 \cdot 9 \\
7 \cdot 5 \\
6 \cdot 7\end{array}$ \\
\hline
\end{tabular}

A, Regular medium; B, concentration of L-glutamic acid, L-proline and L-glutamine quadrupled; $\mathrm{C}$, concentration of $\mathbf{L}$-glutamic acid, only, quadrupled.

* Based on 20 opacity units of bacterial concentration $/ \mathrm{ml}$. in the mouse protection tests; U.S. Standard Pertussis Vaccine contained 8 immunogenic units $/ \mathrm{ml}$.

\section{DISCUSSION}

With respect to the amino acid requirements of phase 1 Bordetella pertussis, a position of primary importance has been assigned to glutamic acid (Rowatt, 1957 $b$; Jebb \& Tomlinson, 1951). Ungar et al. (1950), who used a paper chromatographic technique, observed that alanine, aspartic and glutamic acids, glycine, proline, serine and threonine were used when $B$. pertussis was cultivated in the partially defined liquid medium of Cohen \& Wheeler (1946). In the present work, proline, glutamine and glutamic acid were found to be limiting components for growth of phase I $B$. pertussis in a chemically defined medium, in the presence of a full complement of amino acids. Fukumi, Sayama, Tomizawa \& Uchida (1953) suggested that proline was converted to glutamic acid by $B$. pertussis. Such a conversion may also apply to glutamine. Alanine, glycine, serine and, to a more limited extent, histidine, are utilized by the organism. The use of these components would probably be related, to a great extent, to the availability of glutamic acid, proline and glutamine. The utilization of aspartic acid could not be considered as comparable in importance to that of glutamic acid. The requirement for cystine (cysteine) and glutathione in preference to methionine as sulphur and nitrogen sources may be in part a function of their effect on the redox potential during bacterial growth. The advantage of these particular sulphur-containing components for the nutrition of $B$. pertussis has already been noted (Jebb \& Tomlinson, 1955; Rowatt, 1957b).

Jebb \& Tomlinson (1955), who used small inocula, found that the growth of Bordetella pertussis was consistently better in media prepared with L-amino acids than with the DL-amino acids. The diminished growth observed in our medium when L-acids were replaced by equal weights of DL-amino acid, must have been due to the decrease, by half, of the amount of one or more of the L-amino acids (cystine, glutamic acid, glutamine, proline) present in medium A. It should be noted that B. pertussis used the D-isomers, D-alanine, D-serine and probably $\mathbf{D}$-histidine. $\mathbf{D}$-amino acids (e.g. D-alanine) are known to occur in bacterial cell walls. 
The grouping of a preferential order of amino acids used as a result of metabolism and growth by phase I Bordetella pertussis is shown on Table 1 of the accompanying paper (Vajdic, Goldner \& Wilson, 1966). Omission from the chemically defined medium $\mathbf{A}$ of the ten amino acids shown in the lower level of the order did not adversely affect growth. The addition of increased amounts of glutamic acid, proline and glutamine provided similar maximal yields, whether with or without the full complement of amino acids.

The antigenicity results (Table 3 ) were based only on a small number of protection tests. However, increased amounts of certain amino acids (e.g. glutamic acid) in the original medium (medium A) appeared to increase antigenic potency as well as growth.

The authors wish to thank Dr J. M. Corkill for performing the mouse protection tests, and Drs H. Pivnick and A. H. W. Hauschild for their advice on the manuscript.

\section{REFERENCES}

BLock, R. J. (1950). Estimation of amino acids and amines on paper chromatograms. Anal. Chem. 22, 1327.

Block, R. J., Durrum, E. L. \& Zweig, G. (1958). Quantitative methods. In A Manual of Paper Chromatography and Paper Electrophoresis, pp. 85-109. New York: Academic Press, Inc.

Cohen, S. M. \& Wheeler, M. W. (1946). Pertussis vaccine prepared with phase-1 cultures grown in fluid medium. Am. J. pub. Hlth 36, 371.

Fukumi, H., Sayama, E., Tomizawa, J. \& Uchida, T. (1953). Nutritional requirements and respiratory pattern of pertussis-parapertussis-bronchisepticus group of microorganisms. Jap. J. med. Sci. Biol. 6, 587.

Hanes, C. S., Harris, C. K., Moscarello, M. A. \& Tigane, E. (1961). Quantitative chromatographic methods. Part 4. Stabilized chromatographic systems of high resolving power for amino acids. Can. J. Biochem. Physiol. 39, 163.

JeBb, W. H. H. \& Tombinson, A. H. (1951). The catabolic activity of washed suspensions of Haemophilus pertussis. J. gen. Microbiol. 5, 951.

JEBB, W. H. H. \& Tomlinson, A. H. (1955). The nutritional requirements of Haemophilus pertussis. J. gen. Microbiol. $13,1$.

Rowatr, E. $(1957 a)$. The growth of Bordetella pertussis: a review. J. gen. Microbiol. 17 , 297.

Rowatr, E. $(1957 b)$. Some factors affecting the growth of Bordetella pertussis. J. gen. Microbiol. 17, 279.

Slotta, K. H. \& Primosigh, J. (1951). Amino-acid composition of crotoxin. Nature, Lond. 168, 696.

Ungar, J., James, A. M., Muggleton, P. W., Pegler, H. F. \& Tomich, E. G. (1950). The cultivation of Haemophilus pertussis in partially-defined liquid media. J. gen. Microbiol. 4, 345.

VAJdic, A. H., Goldner, M. \& Wilson, R. J. (1966). The amino acid used in cultures of phase 1 Bordetella pertussis during growth in chemically defined media. J. gen. Microbiol. 44, 445.

Wade, E. H. M., Matheson, A. T. \& Hanes, C. S. (1961). Quantitative chromatographic methods. Part 3. Factors controlling the patterns of separation of the amino acids on paper chromatograms. Can. J. Biochem. Physiol. 39, 141.

Wirson, R. J. (1963) Cultivation of Bordetella pertussis (phase 1) in a chemically-defined medium. Can. J. pub. Hlth 54, 518.

Worcester, J. \& Wrison, E. B. (1943). A table determining LD 50 or the fifty percent end-point. Proc. natn. Acad. Sci., U.S.A. 29, 207. 